\title{
Binary orbits as the driver of $\gamma$-ray emission and mass ejection in classical novae
}

\author{
Laura Chomiuk $^{1}$, Justin D. Linford ${ }^{1}$, Jun Yang ${ }^{2,3,4}$, T. J. O’Brien ${ }^{5}$, Zsolt Paragi ${ }^{3}$, Amy J. Mioduszewski ${ }^{6}$, R. J. Beswick ${ }^{5}$, \\ C. C. Cheung ${ }^{7}$, Koji Mukai ${ }^{8,9}$, Thomas Nelson ${ }^{10}$, Valério A. R. M. Ribeiro ${ }^{11}$, Michael P. Rupen ${ }^{6,12}$, J. L. Sokoloski ${ }^{13}$, \\ Jennifer Weston ${ }^{13}$, Yong Zheng ${ }^{13}$, Michael F. Bode ${ }^{14}$, Stewart Eyres ${ }^{15}$, Nirupam Roy ${ }^{16}$ \& Gregory B. Taylor ${ }^{17}$
}

\begin{abstract}
Classical novae are the most common astrophysical thermonuclear explosions, occurring on the surfaces of white dwarf stars accreting gas from companions in binary star systems ${ }^{1}$. Novae typically expel about $10^{-4}$ solar masses of material at velocities exceeding 1,000 kilometres per second. However, the mechanism of mass ejection in novae is poorly understood, and could be dominated by the impulsive flash of thermonuclear energy ${ }^{2}$, prolonged optically thick winds ${ }^{3}$ or binary interaction with the nova envelope ${ }^{4}$. Classical novae are now routinely detected at gigaelectronvolt $\gamma$-ray wavelengths ${ }^{5}$, suggesting that relativistic particles are accelerated by strong shocks in the ejecta. Here we report high-resolution radio imaging of the $\gamma$-rayemitting nova V959 Mon. We find that its ejecta were shaped by the motion of the binary system: some gas was expelled rapidly along the poles as a wind from the white dwarf, while denser material drifted out along the equatorial plane, propelled by orbital motion ${ }^{6,7}$. At the interface between the equatorial and polar regions, we observe synchrotron emission indicative of shocks and relativistic particle acceleration, thereby pinpointing the location of $\gamma$-ray production. Binary shaping of the nova ejecta and associated internal shocks are expected to be widespread among novae ${ }^{8}$, explaining why many novae are $\gamma$-ray emitters ${ }^{5}$.
\end{abstract}

The identification of the $\gamma$-ray transient J0639+0548, detected by NASA's Fermi Gamma-ray Space Telescope, with the classical nova V959 Mon $^{5}$ was a surprise, because gigaelectronvolt $\gamma$-rays are produced by the inverse Compton mechanism, the pion production mechanism or both ${ }^{9}$, requiring a population of relativistic particles which had not been predicted or observed in normal classical novae. Gigaelectronvolt $\gamma$-rays had been reported from only one nova before V959 Mon, in a system with an unusual Mira giant companion, dense circumbinary material and, thereby, a strong shock interaction between the nova ejecta and the surroundings ${ }^{10}$. The white dwarf in V959 Mon, however, has a main-sequence companion and, therefore, a low-density circumbinary environment ${ }^{11,12}$, and so there is no apparent mechanism for diffusive shock acceleration in an interaction with surrounding material.

The $\gamma$-ray emission from V959 Mon was discovered on 2012 June 19 (day 0 ) and lasted $\sim 12 \mathrm{~d}$, showing a soft-spectrum continuum ${ }^{5}$. Little is known about V959 Mon during the period of $\gamma$-ray emission, owing to its solar conjunction in the first few months of outburst, which prevented optical observations; the transient was not even identified as a nova until $56 \mathrm{~d}$ after $\gamma$-ray discovery ${ }^{5}$. However, we obtained early radio observations coincident with the Fermi detections using the Karl G. Jansky Very Large Array (VLA), just 12 and $16 \mathrm{~d}$ after discovery (Fig. 1).
These observations span a frequency range of $1-6 \mathrm{GHz}$ and show a flat radio spectrum ( $\alpha \approx-0.1$, where $S_{v} \propto v^{\alpha} ; v$ is the observing frequency and $S_{v}$ is the flux density at this frequency). This spectral index is much more consistent with synchrotron radiation than the expected optically thick emission from warm nova ejecta ${ }^{13,14}(\alpha \approx 2$ is predicted and observed in V959 Mon at later times; Fig. 1 and Extended Data Fig. 1).

Like gigaelectronvolt $\gamma$-rays, synchrotron emission requires a population of relativistic particles, and so we can use this radio emission as a tracer of $\gamma$-ray production that lasts longer and enables much higher spatial resolution than do the $\gamma$-rays themselves. The location of the $\gamma$ ray-producing shocks was revealed by milliarcsecond-resolution radio imaging using very-long-baseline interferometric (VLBI) techniques, which are sensitive to high-surface-brightness synchrotron emission. VLBI observations were achieved with the European VLBI Network (EVN) and the Very Long Baseline Array, and spanned 2012 September 18 to October 30 (91-133 d after $\gamma$-ray discovery, 2-7 mas resolution; where one milliarcsecond $(1 \mathrm{mas}) \approx 2 \times 10^{13} \mathrm{~cm}$ at the distance of V959 Mon ${ }^{11}$; Extended Data Table 3). The first VLBI epoch revealed two distinct knots of emission separated by 36 mas, which we subsequently observed to travel away from one another at an estimated rate of $\sim 0.4$ mas d $^{-1}$ (Fig. 2a). In addition, a third radio component appeared in our imaging from day 113 . The brightest component was slightly resolved by the Very Long Baseline Array on day 106 (Extended Data Fig. 2), and had a peak brightness temperature, $\sim 2 \times 10^{6} \mathrm{~K}$, indicative of non-thermal emission (X-ray observations from around this time ${ }^{15}$ imply that hot shocked thermal gas can account for only $<10 \%$ of the radio flux density seen in the VLBI knots; Supplementary Information).

Observations made with the e-MERLIN array (54 mas resolution) just before the first VLBI epoch shows that the compact VLBI components were embedded in a larger-scale structure which was mostly extended east-west (and not detected in the VLBI imaging, because these highresolution arrays with widely separated antennas are not sensitive to such diffuse emission; Fig. 2b). This diffuse emission is interpreted as bremsstrahlung from the bulk of the nova ejecta ${ }^{13,14}$. Whereas the $5 \mathrm{GHz}$ flux density detected in our VLBI imaging was roughly constant or declined with time, the flux density detected in the lower-resolution observations rapidly increased during this period (Fig. 1). The VLBI knots comprised $19 \%$ and $9 \%$ of the total $5 \mathrm{GHz}$ flux density on days 91 and 117 , respectively, implying that over time the synchrotron emission becomes overwhelmed by thermal emission from the warm ejecta. Although synchrotron radio emission has been detected from outbursting novae with red giant companions and dense circumbinary material ${ }^{16-18}$, it has

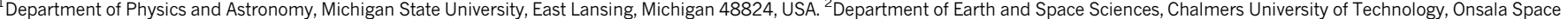

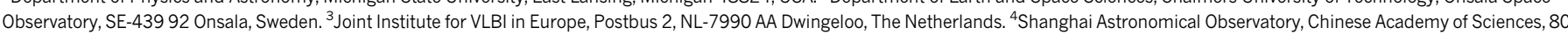

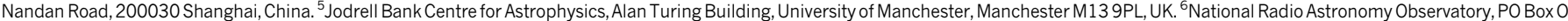

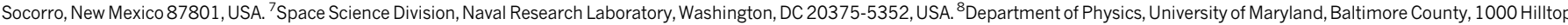

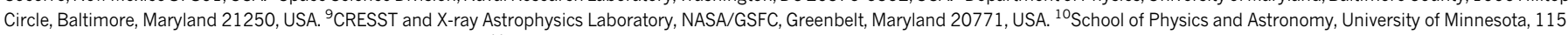

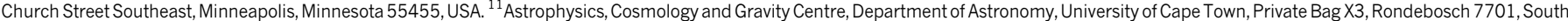
Africa. ${ }^{12}$ National Research Council, Herzberg Astronomy and Astrophysics, 717 White Lake Road, PO Box 248, Penticton, British Columbia V2A 6J9, Canada. ${ }^{13}$ Columbia Astrophysics Laboratory,

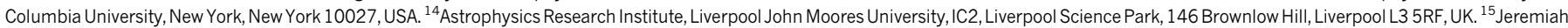
Horrocks Institute for Mathematics, Physics and Astronomy, University of Central Lancashire, Preston PR1 2HE, UK. ${ }^{16}$ Max-Planck-Institut für Radioastronomie, Auf dem Hügel 69, D-53121 Bonn, Germany. ${ }^{17}$ Department of Physics and Astronomy, University of New Mexico, MSC07 4220, Albuquerque, New Mexico 87131-0001, USA.
} 

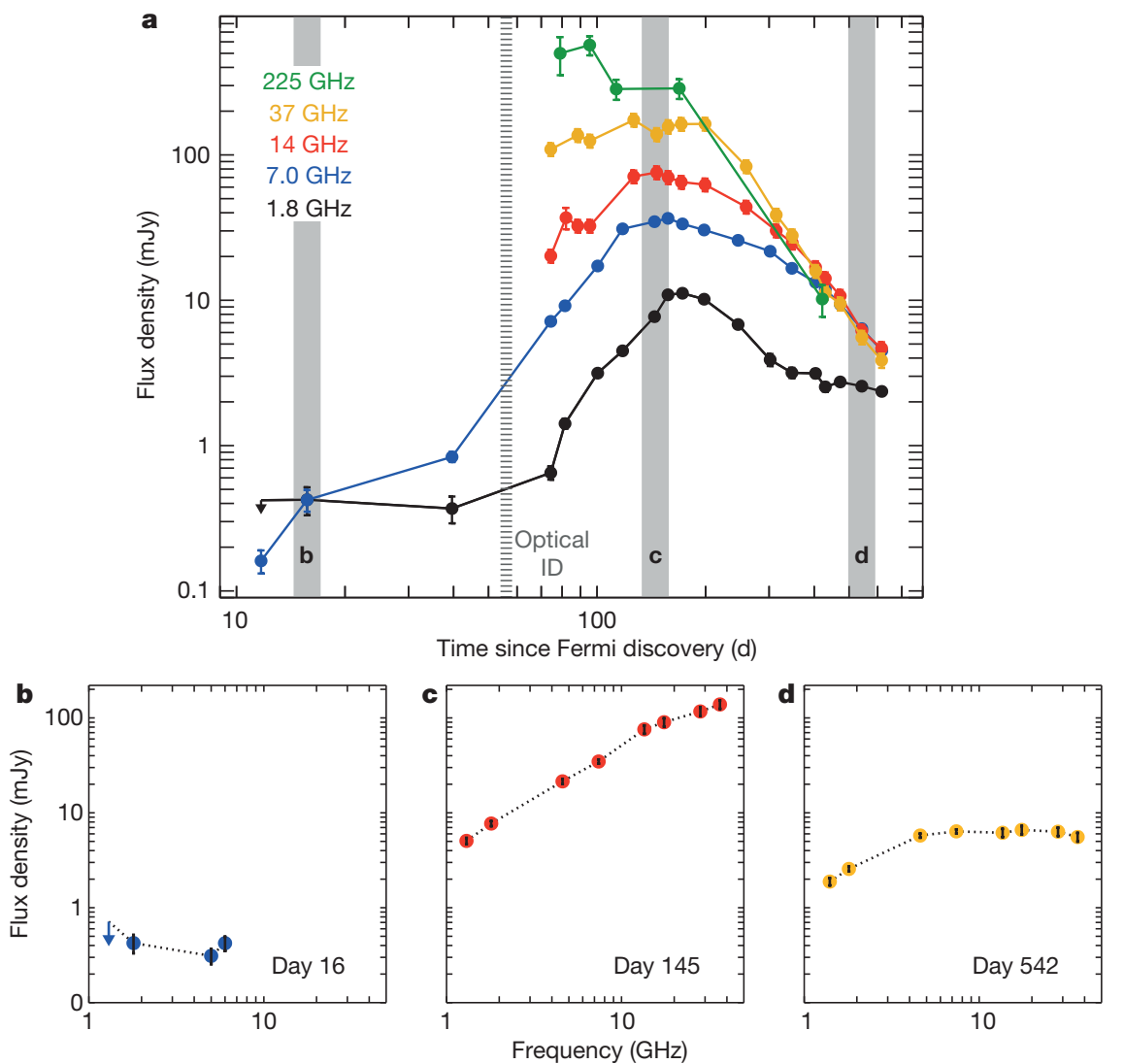

b

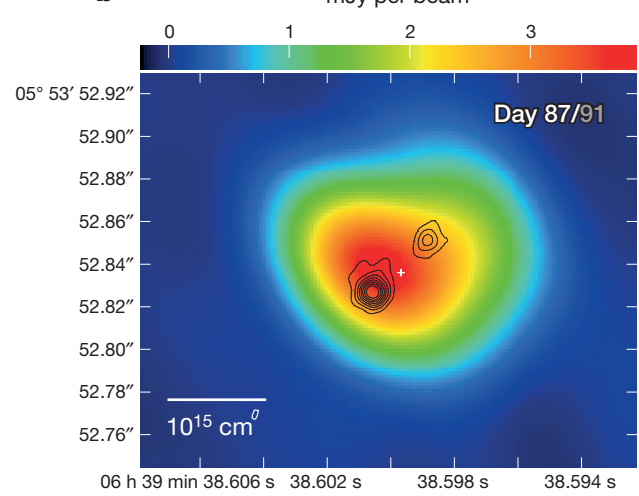

c

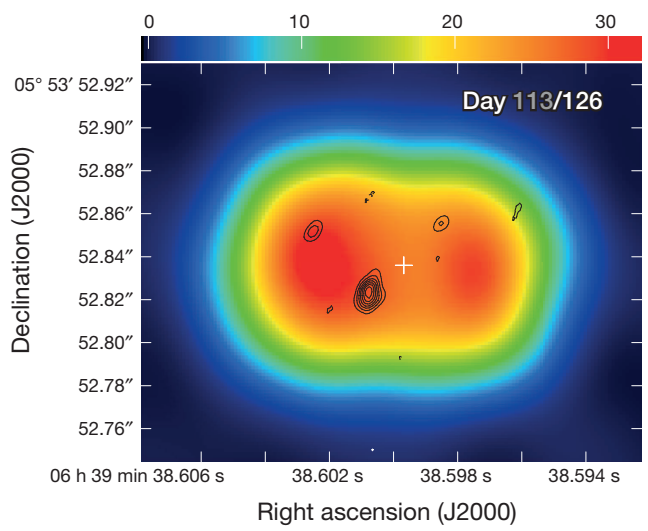

$\begin{array}{lllll}06 \mathrm{~h} 39 \min 38.605 \mathrm{~s} & 38.603 \mathrm{~s} & 38.601 \mathrm{~s} & 38.599 \mathrm{~s} & 38.597 \mathrm{~s}\end{array}$

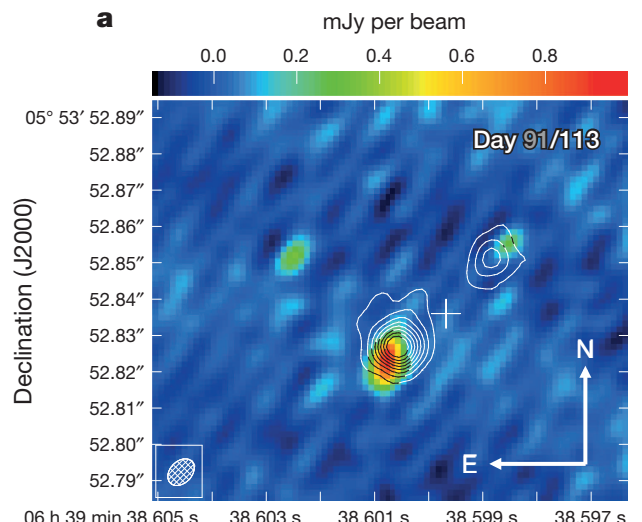

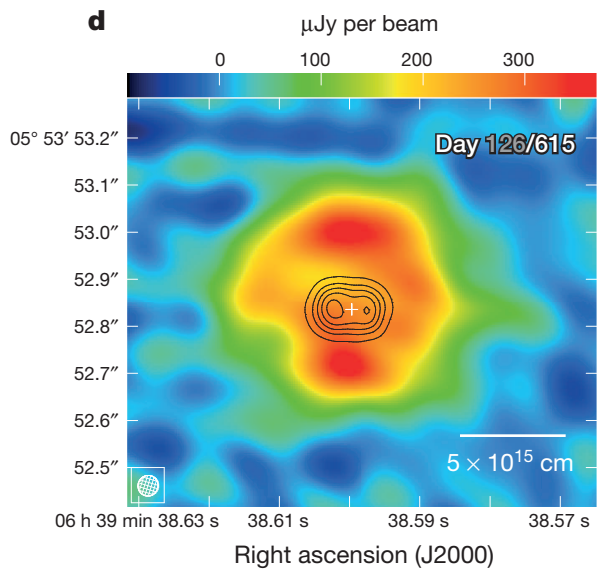

Figure $1 \mid$ Radio light curves and spectra of V959 Mon. a, The multi-frequency light curve (Extended Data Tables 1 and 2) is as expected for expanding thermal ejecta, except at the earliest times $(<30 \mathrm{~d})$ and lowest frequencies $(<2 \mathrm{GHz}$; Supplementary Information). The time of optical identification is marked (hatched line). The times corresponding to three select radio spectra are marked with grey bars. b-d, Early-time flat spectrum (16 d after Fermi discovery; b), transitioning to an optically thick thermal spectrum (day 145; c), and a late thermal spectrum that is mostly optically thin (day 542 ; d). Error bars denote $1 \sigma$ uncertainties. Downward-facing arrows denote $3 \sigma$ upper limits.
Figure $2 \mid$ Radio imaging of V959 Mon. a, Illustration of the expansion of the compact radio knots, with a high-resolution $5 \mathrm{GHz}$ EVN image from $113 \mathrm{~d}$ after $\gamma$-ray discovery shown in colour, and contours representing the EVN image from day 91 . Contour levels span $0.125-2$ mJy per beam in steps of $0.125 \mathrm{mJy}$ per beam. $\mathbf{b}, 5.8 \mathrm{GHz}$ e-MERLIN colour image of thermal nova ejecta on day 87 . The compact VLBI knots from day 91 are superimposed as contours, with levels as in a. c, Components similar to those in $\mathbf{b}$, but one month later, with a $36.5 \mathrm{GHz}$ VLA colour image of the thermal ejecta on day 126. The VLBI knots from day 113 are contours. d, Expansion and flip of the thermal nova ejecta, comparing VLA images from four months and, respectively, two years after outburst. The $17.5 \mathrm{GHz}$ image from day 615 is shown in colour, overplotted with the $36.5 \mathrm{GHz}$ day-126 image now in black contours $(3.2,9.6,16,22.4$ and $28.8 \mathrm{mJy}$ per beam). In all panels, the presumed location of the binary is marked as a white cross. Scale bars in $\mathbf{b}$ and $\mathbf{d}$ assume a distance of $1.5 \mathrm{kpc}$ (ref. 11). Synthesized beams for contours are shown in the bottom left corners of $\mathbf{a}$ and $\mathbf{d}$. White date labels are for colour images; grey labels are for contours. 

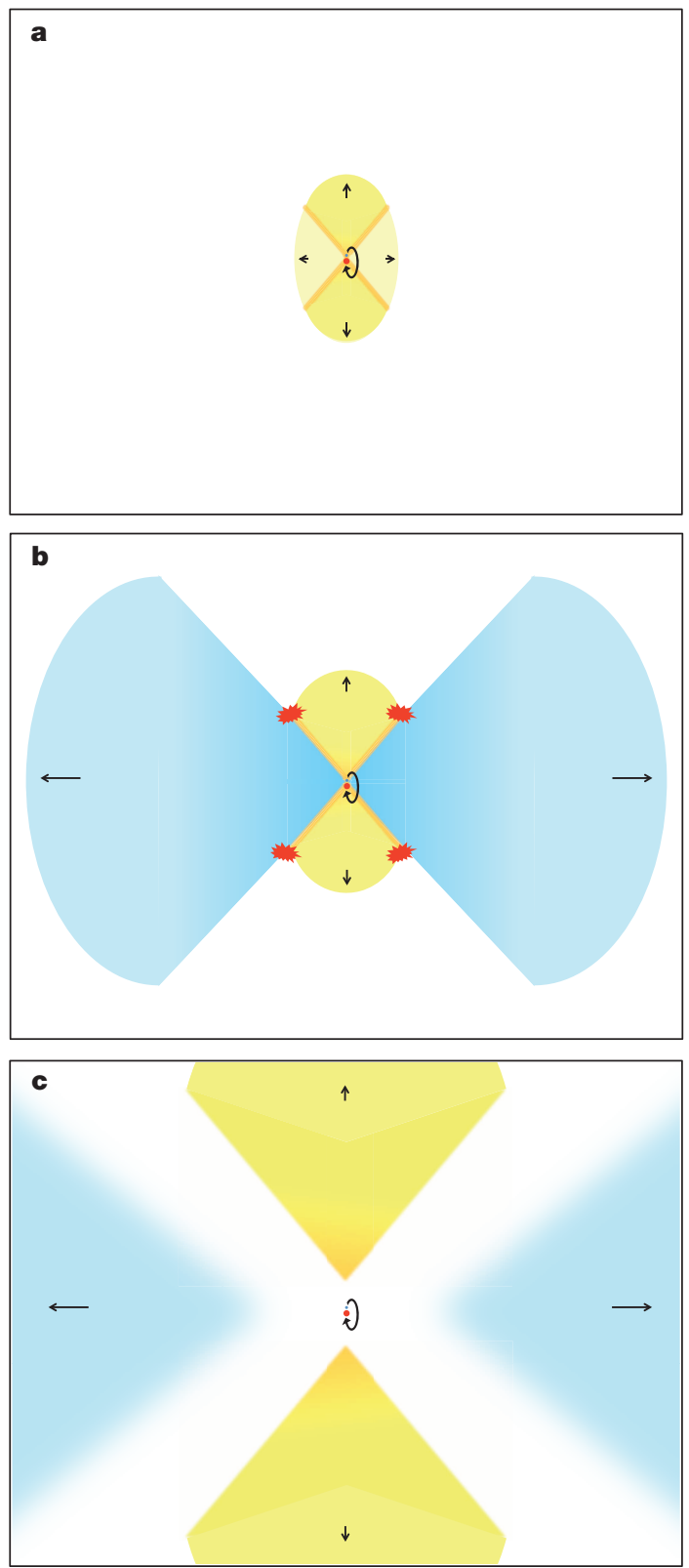

Figure 3 Simple illustration of the 2012 outburst of V959 Mon.

a, Immediately following the thermonuclear runaway, the nova envelope expands (yellow ellipse) and interacts with the binary system, yielding dense material in the equatorial plane ${ }^{22}$ (darker yellow; here orientated vertically). Denser bow shocks surround this puffy equatorial disk ${ }^{7}$ (orange lines). b, As the nova outburst progresses, the white dwarf powers a fast wind ${ }^{3,23}$ that is funnelled towards the low-density poles ${ }^{6,7}$ (blue cones; compare with the thermal emission imaged with VLA on day 126; Fig. 2c). The differential velocity produces shocks ${ }^{8}$ (orange lines), and at the edges of the optically thick ejecta the shocked material yields compact radio knots (red blobs; compare with VLBI knots in Fig. 2). c, Once the white dwarf wind ceases, the polar outflow will detach from the binary and quickly drop in density as it expands (blue cones). The slower-expanding equatorial material will remain dense for longer (yellow regions), and will dominate the radio images at late times (compare with day-615 VLA imaging; Fig. 2d).

not previously been securely identified in novae with main-sequence companions ${ }^{13}$, owing to a paucity of high-resolution radio imaging enabling components of differing surface brightness to be clearly distinguished.

The expanding thermal ejecta were resolved with the VLA when it entered its high-resolution A configuration. An image from 2012 October 23 (day 126; 43 mas resolution) shows that the ejecta have expanded and assumed a clearly bipolar geometry consistent with analyses of optical spectral line profiles ${ }^{19,20}$ (Fig. 2c). The apparent geometry of the ejecta is conveniently simplified, because we view the orbital plane of V959 Mon edge-on ${ }^{21}$. Our imaging illustrates that the VLBI knots were not simple jet-like protrusions from the thermal ejecta. First, there were three VLBI knots when only two are expected from a bipolar jet structure. Second, the major axis of the thermal ejecta (directed east-west) was not well aligned with the expansion of the VLBI knots, but was offset by $45^{\circ}$. In addition, the thermal ejecta expanded faster than the VLBI knots (0.64 mas d $^{-1}$ in diameter; Extended Data Fig. 3). Finally, because the warm thermal ejecta were optically thick at the time of this imaging, the VLBI knots were superimposed around the edges of the ejecta, appearing to surround the two thermal lobes.

The origin of the compact radio knots was clarified when we revisited V959 Mon sixteen months later, when the VLA was next in its A configuration (2014 February 24; day 615; Fig. 2d). The much-expanded thermal ejecta maintained a bi-lobed morphology, but the axis of elongation had rotated so that the brightest regions were oriented northsouth, perpendicular to the outflow observed in 2012. The position angle of the VLBI knots lay roughly halfway between that of the early and late axes of ejecta expansion (Fig. 2).

This apparent rotation of the thermal ejecta between day 126 and day 615 was due to the outflow being faster along the east-west axis, with the result that the east-west lobes became optically thin first. Just such an asymmetry is predicted by hydrodynamic simulations of interacting winds shaped by orbital evolution ${ }^{6}$. In this scenario, binary stars orbiting within the nova envelope transfer some of their orbital energy to the surrounding material through viscous interaction, thereby expelling the envelope preferentially along the orbital plane $e^{22,23}$ (Fig. 3a), corresponding to a north-south orientation in V959 Mon. This equatorial material is observable as thermal ejecta, but it expands relatively slowly, and its compact structure therefore proves difficult to image at early times. Meanwhile, a fast, prolonged wind is blown off the white dwarf ${ }^{3}$, and this thermal wind preferentially expands in the low-density polar directions ${ }^{7}$ (Fig. 3b). At early times, while the ejecta are optically thick, this fast material expanding along the poles will dominate the radio images, as in Fig. 2c. Later, when the thermal radio emission becomes optically thin, the dense material in the orbital plane will be brightest (Figs $2 \mathrm{~d}$ and 3c). A similar $90^{\circ}$ flip of the major axis has been hinted at in radio imaging of other novae $e^{24-26}$, suggesting that such a transformation may be common in classical novae.

The VLBI knots, and, by extension, the $\gamma$-ray emission, appear to be produced in the interaction between the rapidly expanding material driven along the poles and the slower equatorial material (Fig. 3c). This interaction within the ejecta could explain the prolonged duration of the radio synchrotron emission ${ }^{8}$, which lasts as long as the fast wind flows past the dense material concentrated in the orbital plane.

The mass ejection observed in V959 Mon is a version of the commonenvelope phase that occurs in all close binary stars, and is a critical step in the formation of diverse phenomena like X-ray binaries, type Ia supernovae and stripped-envelope supernovae. Despite its widespread significance, common-envelope evolution remains one of the most poorly understood phases of binary evolution, with few observational tests and models that often fail to expel the envelope at all ${ }^{27,28}$. V959 Mon shows that classical novae can serve as a test-bed for developing an understanding of common-envelope evolution, and that common-envelope interaction has a role in the ejection of nova envelopes.

An extensive multi-wavelength observational campaign shows V959 Mon to be a typical classical nova. Its expansion velocities, spectral line profiles, binary period, binary companion and optical light curve fall well within expected ranges ${ }^{11,19-21}$. Additionally, after the few early epochs showing a flat radio spectrum, the radio light curve of V959 Mon became consistent with thermal emission from expanding warm ejecta, implying $4 \times 10^{-5}$ solar masses of ejecta (a typical value for a classical nova ${ }^{1}$; Extended Data Fig. 4 and Supplementary Information). The only unusual characteristic of V959 Mon is its proximity; at a distance of $\lesssim 2 \mathrm{kpc}$ (ref. 11), it is several times closer than the typical nova which explodes 
in the Galactic bulge ( $~ 8 \mathrm{kpc}$ distant). Therefore, $\gamma$-rays could be a common feature of normal classical novae.

Since the outburst of V959 Mon, three additional classical novae have been identified with Fermi $^{5,29}$, further implying that V959 Mon is not unusual, and many novae produce $\gamma$-rays. The recent increase in the rate of Fermi detections of novae can probably be explained by a combination of deeper, targeted detection efforts and a lucky crop of nearby novae; with effort, the sample of $\gamma$-ray-detected novae will continue to grow. The mechanism we propose here for powering the $\gamma$-ray emission in V959 Mon-binary interaction shaping nova ejecta and powering strong internal shocks-works in most novae, implying that each of these garden-variety explosions accelerates particles to relativistic speeds.

Online Content Methods, along with any additional Extended Data display items and Source Data, are available in the online version of the paper; references unique to these sections appear only in the online paper.

Received 23 June; accepted 12 August 2014.

Published online 8 October 2014

1. Gehrz, R. D., Truran, J. W., Williams, R. E. \& Starrfield, S. Nucleosynthesis in classical novae and its contribution to the interstellar medium. Publ. Astron. Soc. Pacif. 110, 3-26 (1998).

2. Starrfield, S., Truran, J. W. Sparks, W. M. \& Kutter, G. S. CNO abundances and hydrodynamic models of the nova outburst. Astrophys. J. 176, 169-176 (1972).

3. Kato, M. \& Hachisu, I. Optically thick winds in nova outbursts. Astrophys. J. 437, 802-826 (1994).

4. MacDonald, J. The effect of a binary companion on a nova outburst. Mon. Not. R. Astron. Soc. 191, 933-949 (1980)

5. The Fermi-LAT collaboration. Fermi establishes classical novae as a distinct class of $\gamma$-ray sources. Science 345, 554-558 (2014).

6. Soker, N. \& Livio, M. Interacting Winds and the shaping of planetary nebulae. Astrophys. J. 339, 268-278 (1989)

7. Porter, J. M., O'Brien, T. J. \& Bode, M. F. On the asphericity of nova remnants caused by rotating white dwarf envelopes. Mon. Not. R. Astron. Soc. 296, 943-948 (1998)

8. Shankar, A., Livio, M. \& Truran, J. W. The common envelope phase in classical novae: one-dimensional models. Astrophys. J. 374, 623-630 (1991).

9. Dubus, G. Gamma-ray binaries and related systems. Astron. Astrophys. Rev. 21, 64 (2013).

10. Abdo, A. A. et al. Gamma-ray emission concurrent with the nova in the symbiotic binary V407 Cygni. Science 329, 817-821 (2010)

11. Munari, U. et al. Photometric evolution, orbital modulation and progenitor of Nova Mon 2012. Mon. Not. R. Astron. Soc. 435, 771-781 (2013).

12. Hoard, D. W. et al. Nova-like cataclysmic variables in the infrared. Astrophys. J. 786, 68 (2014).

13. Seaquist, E. R. \& Bode, M. F. in Classical Novae (eds Bode, M. F. \& Evans, A.) 141-166 (Cambridge Univ. Press, 2008)

14. Roy, N. et al. Radio studies of novae: a current status report and highlights of new results. Bull. Astron. Soc. India 40, 293-310 (2012).

15. Nelson, T. et al. X-ray and UV observations of Nova Mon 2012. Astron. Telegram 4321 (2012)

16. Seaquist, E. R. et al. A detailed study of the remnant of nova GK Persei and its environs. Astrophys. J. 344, 805-825 (1989).

17. O'Brien, T. J. etal. An asymmetric shock wave in the 2006 outburst of the recurrent nova RS Ophiuchi. Nature 442, 279-281 (2006).
18. Kantharia, N. G. et al. Rapid rise in the radio synchrotron emission from the recurrent nova system V745 Sco. Astron. Telegram 5962 (2014).

19. Ribeiro, V. A. R. M., Munari, U. \& Valisa, P. Optical morphology, inclination, and expansion velocity of the ejected shell of Nova Monocerotis 2012. Astrophys. J. 768, 49 (2013).

20. Shore, S. N. et al. The spectroscopic evolution of the $\gamma$-ray emitting classical nova Nova Mon 2012. I. Implications for the ONe subclass of classical novae. Astron Astrophys. 553, A123 (2013).

21. Page, K. L. et al. The $7.1 \mathrm{hr} X$-ray-ultraviolet-near-infrared period of the $\gamma$-ray classical Nova Monocerotis 2012. Astrophys. J. 768, L26 (2013).

22. Livio, M., Shankar, A., Burkert, A. \& Truran, J. W. The common envelope phase in the outbursts of classical novae. Astrophys. J. 356, 250-254 (1990)

23. Lloyd, H. M., O'Brien, T.J. \& Bode, M. F. Shaping of nova remnants by binary motion. Mon. Not. R. Astron. Soc. 284, 137-147 (1997)

24. Taylor, A. R., Hjellming, R. M., Seaquist, E. R. \& Gehrz, R. D. Radio images of the expanding ejecta of nova QU Vulpeculae 1984. Nature 335, 235-238 (1988).

25. Eyres, S. P. S., Davis, R. J. \& Bode, M. F. Nova Cygni 1992 (V1974 Cygni): MERLIN observations from 1992 to 1994. Mon. Not. R. Astron. Soc. 279, 249-256 (1996).

26. Heywood, I., O'Brien, T. J., Eyres, S. P. S., Bode, M. F. \& Davis, R. J. V723 Cas (Nova Cassiopeiae 1995): MERLIN observations from 1996 to 2001. Mon. Not. R. Astron Soc. 362, 469-474 (2005).

27. Passy, J. et al. Simulating the common envelope phase of a red giant using smoothed-particle hydrodynamics and uniform-grid codes. Astrophys. J. 744, 52 (2012).

28. Ivanova, N. et al. Common envelope evolution: where we stand and how we can move forward. Astron. Astrophys. Rev. 21, 59 (2013).

29. Cheung, C. C., Jean, P. \& Shore, S. N. Fermi-LAT $\gamma$-ray observations of Nova Centauri 2013. Astron. Telegram 5649 (2013).

Supplementary Information is available in the online version of the paper.

Acknowledgements The National Radio Astronomy Observatory (NRAO) is a facility of the US National Science Foundation (NSF) operated under cooperative agreement by Associated Universities, Inc. The EVN is a joint facility of European, Chinese, South African and other radio astronomy institutes funded by their respective national research councils. The EVN and e-VLBI research infrastructures were supported by the European Commission Seventh Framework Programme (FP/2007-2013) under grant agreements nos 283393 (RadioNet3) and RI-261525 (NEXPReS). e-MERLIN is operated by The University of Manchester at Jodrell Bank Observatory on behalf of the Science and Technology Facilities Council. The SMA is a joint project between the Smithsonian Astrophysical Observatory and the Academia Sinica Institute of

Astronomy and Astrophysics. Support for CARMA construction came from the Moore Foundation, the Norris Foundation, the McDonnell Foundation, the Associates of the California Institute of Technology, the University of Chicago, the states of California, Illinois and Maryland, and the NSF. Ongoing CARMA development and operations are supported by the NSF and by the CARMA partner universities. L.C. is a Jansky Fellow of the NRAO. This research received funding from NASA programmes DPR S-15633-Y and 10-FERMI10-C4-0060 (C.C.C.), NASA award NNX13A091G (T.N.), NSF award AST-1211778 (J.L.S. and J.W.), the South African SKA Project (V.A.R.M.R.) and the Alexander von Humboldt Foundation (N.R.)

Author Contributions L.C. wrote the text. L.C., J.D.L., J.Y., T.J.O., Z.P., A.J.M., C.C.C., R.J.B., T.N., Y.Z., J.W. and G.B.T. obtained and reduced the data. All authors contributed to the interpretation of the data and commented on the final manuscript.

Author Information Reprints and permissions information is available at www.nature.com/reprints. The authors declare no competing financial interests. Readers are welcome to comment on the online version of the paper. Correspondence and requests for materials should be addressed to L.C. (chomiuk@pa.msu.edu). 


\section{METHODS}

VLA. We observed V959 Mon with the VLA from Jun $2012^{30}$ until the time of publication as part of programmes S4322, 12B-375, 13A-461, 13B-057 and S61420. Monitoring was carried out in the $\mathrm{L}(1-2 \mathrm{GHz}), \mathrm{C}(4-8 \mathrm{GHz}), \mathrm{Ku}(12-18 \mathrm{GHz})$, and $\mathrm{Ka}$ $(26-36 \mathrm{GHz})$ bands across all array configurations ${ }^{31}$. In the $\mathrm{C}, \mathrm{Ku}$ and Ka bands, we observed with two sidebands, each composed of eight spectral windows with 64 $2 \mathrm{MHz}$-wide channels and four polarization products. Each sideband had a bandwidth of $1 \mathrm{GHz}$, and the two sidebands were separated to enhance our frequency coverage. In the L band, we obtained only $1 \mathrm{GHz}$ of bandwidth in total, covering the entire available frequency range in this band.

In the first three epochs, the entire positional error circle of the Fermi transient was observed (95\% confidence radius of $11 \mathrm{arcmin}$ ), with a single pointing in the $\mathrm{L}$ band and a seven-pointing mosaic in the $\mathrm{C}$ band. Later, after identification of the optical nova, a single pointing was centred on V959 Mon, typically yielding $\sim 10$ 15 min on source in each band and epoch.

We observed $\mathrm{J} 0632+1022$ as the secondary phase calibrator in the $\mathrm{L}$ and $\mathrm{C}$ bands, and $0643+0857$ in the Ku and Ka bands. 3C147 was used as an absolute flux density and band-pass calibrator. Data were reduced using standard routines in CASA and AIPS, and in most cases a single round of phase-only self-calibration was implemented with 1 min solution intervals. Imaging was carried out in $\mathrm{CASA}^{32}$, AIPS $^{33}$ and Difmap ${ }^{34}$, typically using a Briggs robust value ${ }^{35}$ of 1 . Flux densities were obtained by fitting a Gaussian to the source, using JMFIT in AIPS or gaussfit in CASA. Measurements are presented in Extended Data Table 1. Uncertainties include a $5 \%$ calibration error in the $\mathrm{L}$ and $\mathrm{C}$ bands and a $0 \%$ calibration error in the $\mathrm{Ku}$ and Ka bands.

In the A configuration epochs, which provide the highest angular resolution, special attention was devoted to imaging. To achieve the highest possible resolution, the VLA images shown in Fig. 2 were produced in Difmap with uniform weighting. The $36.5 \mathrm{GHz}$ image from 2012 October 23 featured in Fig. 2c has a synthesized beam of 44 mas $\times 42$ mas full-width at half-maximum (FWHM) and a root mean squared (r.m.s.) sensitivity of $73 \mu \mathrm{Jy}$ per beam. The $17.5 \mathrm{GHz}$ image from 2014 February 24 in Fig. 2 d has a $0.125^{\prime \prime} \times 0.09^{\prime \prime}$ FWHM synthesized beam and r.m.s. noise of $19 \mu$ Jy per beam. When smoothed to similar resolution, images at other frequencies show similar structure on this date. We note that during the first A configuration (October 2012-January 2013), the nova was not spatially resolved at the lower frequencies (L and C bands). In the second A configuration (February 2014), V959 Mon was slightly resolved in the $\mathrm{C}$ band and remained unresolved in the $\mathrm{L}$ band.

Millimetre data. The $225 \mathrm{GHz}$ flux density of V959 Mon was monitored using the Submillimetre Array (SMA) and Combined Array for Research in Millimeter-wave Astronomy (CARMA). Under SMA programme 2012A-S016, observations were obtained from September to December 2012. CARMA observations were obtained at 96 and $230 \mathrm{GHz}$ during May and August 2013 under programme c1130. Data were reduced using standard routines in MIRIAD ${ }^{36}$, and measurements are listed in Extended Data Table 2. Uncertainties include calibration errors of at least $10 \%$.

In addition, we used high-frequency measurements obtained with the Institute for Radio Astronomy in the Millimeter Range (IRAM) $30 \mathrm{~m}$ telescope and the Plateau de Bure Interferometer (PdBI) ${ }^{37}$ (Extended Data Table 2). These measurements additionally constrained our radio/millimetre spectrum fitting and light curve modelling, as shown in Extended Data Figs 1 and 4.

e-MERLIN. The e-MERLIN array was used to observe V959 Mon on a number of occasions, starting in September 2012, when the VLA detected an increase in radio brightness. The first epoch (Fig. 2b) combined observations from 2012 September 13 and 14. The observations used six telescopes of the e-MERLIN array. They were made in the $\mathrm{C}$ band, centred at $5.75 \mathrm{GHz}$ with a bandwidth of $512 \mathrm{MHz}$ per polarization. This total bandwidth was split into four adjacent, $128 \mathrm{MHz}$-wide sub-bands and correlated with 512 channels per sub-band. We observed J0645+0541 as a phase calibrator, 3C286 as a flux calibrator and OQ208 as a band-pass calibrator. Data were calibrated and reduced with standard tasks in AIPS. The data were phase selfcalibrated, and imaging was carried out in AIPS using a Briggs robust parameter of 1 . The synthesized beam used to restore the image is 65 mas $\times 45$ mas. The peak flux density in this map is $3.7 \mathrm{mJy}$ per beam, with r.m.s. noise of $53 \mu \mathrm{Jy}$ per beam. The total flux density is estimated as $7.0 \pm 0.5 \mathrm{mJy}$ (obtained via Gaussian fitting to the image data), which is consistent with estimates made from VLA observations on shorter baselines around the same time.

EVN. We performed the EVN observations of V959 Mon in the C band $(5.0 \mathrm{GHz})$ in five epochs spanning September 2012 to January 2013. Observations were carried out with a data rate of $1,024 \mathrm{Mbit} \mathrm{s}^{-1}$ and 2-bit sampling, yielding dual polarization and a $128 \mathrm{MHz}$ bandwidth. Observation duration was $\sim 7 \mathrm{~h}$ per epoch. The first three observations were EVN Target of Opportunity experiments during the e-EVN sessions (project codes RO005 and RO006), and the last two epochs were part of project EO011. Participating EVN stations were Effelsberg, the phased array of the Westerbork Synthesis Radio Telescope, Onsala, Medicina, Noto, Torun, and the Lovell telescope; Effelsberg was not functioning during the last epoch owing to snow. Through the wideband internet connection to the EVN stations, all observations were correlated in real time at the Joint Institute for VLBI in Europe with the following default correlation parameters: $2 \mathrm{~s}$ integration time and 32 frequency points per sub-band.

We observed J0645+0541 as the phase-referencing source in all our epochs. The J2000 coordinates were RA $=06 \mathrm{~h} 45 \mathrm{~min} 47.27653 \mathrm{~s}$ and dec. $=05^{\circ} 41^{\prime} 22.3857^{\prime \prime}$ (absolute $1 \sigma$ positional uncertainty: 1.1 mas in RA and 2.0 mas in dec.). The separation between the nodding calibrator and V959 Mon was $1.54^{\circ}$. We also observed a secondary calibrator as an astrometric check source, which implied a $1 \sigma$ systematic position error of 0.43 mas in RA and 0.70 mas in dec. We took a nodding cycle time of $1 \mathrm{~min}$ for the calibration and $3 \mathrm{~min}$ for the target.

Initial data calibration was carried out in AIPS using standard routines. After this calibration, the data were averaged in each sub-band. Imaging and deconvolution were then carried out in Difmap. To remove the phase error associated with the source structure, we first imaged the reference source J0645+0541 and then redid fringe-fitting with the calibrator image. The source $06645+0541$ showed a single side core-jet structure with a total flux density of $175 \pm 18 \mathrm{mJy}$. The centroid of the radio core was determined by Gaussian model fitting and later used as the image reference origin. We imaged V959 Mon with natural weighting in Difmap.

The intensity image in the first epoch (2012 September 18) had a synthesized beam with FWHM 9.2 mas $\times 5.5$ mas at a position angle of $-38.0^{\circ}$ and an r.m.s. sensitivity of $0.03 \mathrm{mJy}$ per beam. A pair of knots was clearly detected ${ }^{38}$ (Extended Data Table 3 ). Here we name the brighter (eastern) one A and the weaker (western) one B; this image is shown as white contours in the top panel of Fig. 2. Knots A and B had an angular separation of $35.5 \pm 0.2$ mas. After the knots had been fitted with pointsource models, there still existed some extended emission with a peak brightness of $0.27 \mathrm{mJy}$ per beam in the residual map. We modelled the residual extended emission with two circular Gaussian components (respective FWHMs of 16.4 and 29.9 mas). Each knot was associated with one extended emission region.

We detected the pair of radio knots again in the second epoch (2012 October 10), along with a new, third knot (Fig. 2a). Knots $A$ and $B$ had faded since the first epoch (Extended Data Table 3). Knot A was resolved and its intensity distribution was well fitted by two point sources (A1 and A2) with a separation of $5.7 \pm 0.2$ mas at position angle $-179.5^{\circ}$. The separation was $45.2 \pm 0.4$ mas at position angle $131.9^{\circ}$ between $A 1$ and $B$, and was $49.1 \pm 0.4$ mas at position angle $137.0^{\circ}$ between $A 2$ and $B$. The new, third knot, dubbed $\mathrm{C}$, was located east of $\mathrm{A}$, and was brighter than knot B in this epoch. After fitting and removing the knots, there was some residual large-scale structure showing as regular stripes, most probably a hint of the extended emission seen with the VLA. The synthesized beam had a FWHM of 8.4 mas $\times 6.0$ mas at a position angle of $-44.3^{\circ}$

We find that radio knots A and B both appeared to be moving away from a central position. Between 2012 September 18 and 2012 October 10, they were measured to recede from one another at $0.50 \mathrm{mas} \mathrm{d}^{-1}$.

We failed to detect any compact features associated with V959 Mon in three EVN epochs (2012 November 14, 2012 December 4 and 2013 January 15), because of its low peak brightness and significant expansion. We place $5 \sigma$ upper limits on the peak flux density from compact knots of $<0.25 \mathrm{mJy}$ per beam on 2012 November $14,<0.15$ mJy per beam on 2012 December 4 and $<0.18$ mJy per beam on 2013 January 15. There was a hint of extended emission in the raw image, but it was not possible to locate the position, owing to strong side lobes of the synthesized beam. The source was only weakly detected on the shortest and the most sensitive baseline (Effelsberg-Westerbork) in the third and fourth epochs. V959 Mon was obscured by noise in the last EVN epoch.

Positions and fluxes of the EVN components are listed in Extended Table 3.

Very Long Baseline Array. We observed V959 Mon with the Very Long Baseline Array (VLBA) on 2012 October 3, October 14, October 30 and November 17 under the NRAO project code BM0385. For the first three epochs, we observed in both the $\mathrm{L}$ and the $\mathrm{C}$ band, and the final epoch was in the $\mathrm{C}$ band. Each observation had eight spectral windows covering a total bandwidth of $256 \mathrm{MHz}$ via the new $\mathrm{ROACH}$ digital backend (RDBE) and yielded a total on-source time of $\sim 100 \mathrm{~min}$ per frequency band. For the first epoch, each spectral window had eight channels with $4 \mathrm{MHz}$ of bandwidth per channel. For the subsequent observations, we had 64 channels per spectral window with $500 \mathrm{kHz}$ per channel. The first three epochs had centre frequencies of 1.55 and $4.98 \mathrm{GHz}$ for the $\mathrm{L}$ and $\mathrm{C}$ bands, respectively. The final C-band epoch had a centre frequency of $4.24 \mathrm{GHz}$. The C-band phase reference source was J0650+0358 for the first two epochs and J0645+0541 for the final two epochs (changed to match the calibrator used for EVN observations). The L-band phase reference source was $0650+0358$ for all epochs. The data were reduced using standard routines in AIPS, and images were made using both AIPS and Difmap.

In our first VLBA epoch, we detected VLBI knots A and B in both the $5.0 \mathrm{GHz}$ data and $1.6 \mathrm{GHz}$ data. For the $5.0 \mathrm{GHz}$ image $(3.5$ mas $\times 1.5$ mas synthesized beam; r.m.s. sensitivity, $27 \mu \mathrm{Jy}$ per beam), knot A was not well fitted with a point source and was therefore modelled with a circular Gaussian, whereas knot B was well fitted 
by a point-source model (Extended Data Table 3). We also noticed a possible third radio knot approximately 7.7 mas north of the brightest component. For the $1.6 \mathrm{GHz}$ image ( 11 mas $\times 5$ mas synthesized beam; r.m.s. sensitivity, $49 \mu$ Jy per beam), both component $\mathrm{A}$ and component $\mathrm{B}$ were diffuse and modelled with circular Gaussians. There was no sign of the third component in the $1.6 \mathrm{GHz}$ image.

For the second VLBA epoch, we detected the three EVN knots in the $5.0 \mathrm{GHz}$ image ( 3.8 mas $\times 1.7$ mas synthesized beam; r.m.s. sensitivity, $37 \mu$ Jy per beam). Knots $A$ and $B$ had faded with respect to the first VLBA observation, and knot $C$ was seen to the east of knot A. Knot A was best fitted by two point-source models and a circular Gaussian component to account for diffuse emission. Knots B and C were well fitted by single point sources. In the $1.6 \mathrm{GHz}$ data $(12$ mas $\times 6$ mas synthesized beam; r.m.s. sensitivity, $27 \mu \mathrm{Jy}$ per beam), only knot B was obvious. It was again fitted with a circular Gaussian. There was a second small knot in the vicinity of knot A, and this was also fitted with a circular Gaussian, but we do not believe it accounts for the total flux in knot A.

Unfortunately, the phase reference source did not produce strong fringes on the longer baselines at $5.0 \mathrm{GHz}$ for the third and fourth VLBA epochs. In addition, one antenna was inoperative during each of these observations. The reduced sensitivity and lower resolution led to problems identifying distinct components, and the change in phase reference source made it difficult to compare positions between the first and last two epochs. For the third epoch at $5.0 \mathrm{GHz}$, knot A had a flux density of $1.0 \pm 0.1 \mathrm{mJy}$, whereas knot $\mathrm{B}$ was not detected with high confidence (upper limit of $0.14 \mathrm{mJy}$ ). At $1.6 \mathrm{GHz}$, only one component in the vicinity of knot A was detected unambiguously, but with a dramatically increased flux density of $1.2 \pm 0.1 \mathrm{mJy}$. Knot B had an upper limit of $0.14 \mathrm{mJy}$. The increase in flux density for knot $\mathrm{A}$ and the drop in flux density for knot B could indicate that the shock associated with knot $\mathrm{A}$ encountered a region of higher density leading to increased synchrotron emission, and that knot B exhausted its supply of relativistic particles. For the fourth epoch no $1.6 \mathrm{GHz}$ data were obtained. The source had dimmed significantly at $5.0 \mathrm{GHz}$, and knot A was just barely detected, with a flux density of $0.16 \pm 0.05 \mathrm{mJy}$. Knot B was not detected (upper limit of $0.11 \mathrm{mJy}$ ).

Positions and fluxes of the VLBA components are listed in Extended Data Table 3. We note that some small disagreement between EVN and VLBA positions is expected owing to the use of different phase reference sources.

Concurrent observations at 1.6 and $5.0 \mathrm{GHz}$ enable the creation of a spectral index map. For the 2012 October 3 observations, we applied a $(u, v)$-taper to the $5.0 \mathrm{GHz}$ data to match resolution with our $1.6 \mathrm{GHz}$ image. The matched-resolution images were then aligned via two-dimensional cross correlation, and low signal-to-noise regions were blanked. We used the AIPS task SPIXR to produce the map presented in Extended Data Fig. 2. Knot A had an overall positive spectral index, with a mean $\alpha$ of $\sim 1.2$ in the region of high signal-to-noise ratio. Knot $B$ had an overall negative spectral index, with a mean value of $\alpha \approx-0.3$ in the regions of highest signal-to-noise ratio. The spectra were generally flatter ( $\alpha$ closer to 0 ) near the edges of the knots.

The spectral index of knot $B$ appears roughly constant between days 106 and 117 (Extended Data Table 3). Curiously, knot A appeared to have faded at low frequency on day 117 (leading to $\alpha>1.2$ ), but then brightened drastically at $1.6 \mathrm{GHz}$ on day 133 (yielding $\alpha \approx 0$ ). The optical depth of knot $A$ therefore appears to be time variable.

From our two epochs of $5 \mathrm{GHz}$ data, we can get an independent estimate of the expansion rate for knots A and $\mathrm{B}$. As stated previously, the EVN observations exhibited an expansion rate of $\sim 0.50$ mas $^{-1}$ between 2012 September 18 and October 10. Using the VLBA positions listed in Extended Data Table 3, we find an expansion rate of $\sim 0.35 \mathrm{mas} \mathrm{d}^{-1}$ between 2012 October 3 and October 14 . Because the VLBA observations were made later than the EVN observations, the lower rate may indicate that the expansion was slowing down. However, possible evolution of the source structure and the different $(u, v)$ coverages of the instruments also introduce significant uncertainties into this comparison. Because we have only two epochs with detections in each array, it proves difficult to establish the detailed evolution of the VLBI knot expansion velocity.

Expansion of the thermal ejecta: days 127-199. During the first period of VLA A configuration, we obtained five epochs spanning 2012 October 23-2013 January 4 (Extended Data Table 1). V959 Mon was clearly resolved at frequencies of 13.5$36.5 \mathrm{GHz}$. V959 Mon was bright during this period, and imaged at significance levels of $>100 \sigma$ per synthesized beam. In each image, V959 Mon was fitted with a Gaussian using the task JMFIT in AIPS. The width and position angle of the Gaussian were allowed to vary, and the angular dimensions of V959 Mon were found by deconvolving the synthesized beam from the fitted Gaussian.

Gaussian fits provide simple first-order estimates of the changing dimensions of V959 Mon, although the source is not perfectly described with this profile form (future work will involve a more detailed analysis of the source geometry). The position angles of the fitted Gaussians were constant in time, consistent across frequency $\left(\sim 87^{\circ}\right)$ and distinct from the position angle of the synthesized beams. V959 Mon obviously expanded along both its major (east-west) and minor (north-south) axes over the three months of A-configuration observations. Extended Data Fig. 3 shows the deconvolved radii of V959 Mon over this period, with the semi-major axis plotted in the top panel and the semi-minor axis plotted in the bottom panel. Both axes were observed to increase with remarkable linearity at all four frequencies; linear fits are overplotted. At $13.5 \mathrm{GHz}$, we find diameter expansion rates of $0.62 \mathrm{mas} \mathrm{d}^{-1}$ and 0.64 mas d $^{-1}$ along the minor and major axes, respectively. At $36.5 \mathrm{GHz}$, we find diameter expansion rates of 0.66 mas d $^{-1}$ along the minor axis and 0.37 mas d$^{-1}$ for the major axis.

At any given epoch, very similar minor-axis radii were measured at all four frequencies. However, the size of the major axis varied with frequency, with the exception of the first epoch. Measurements at $36.5 \mathrm{GHz}$ of the major axis yielded systematically smaller radii than did $13.5 \mathrm{GHz}$ measurements (and data points at the intermediate frequencies of 17.5 and $28.2 \mathrm{GHz}$ also follow this trend). Assessing all five epochs, it is clear that the major axis of V959 Mon grew most slowly at highest frequency.

The material expanding along V959 Mon's minor axis was consistent with being optically thick throughout the A-configuration observations. When optically thick, all frequencies display roughly the same radius of the $\tau \approx 1$ surface, as we observed for material expanding along the minor axis of V959 Mon. However, the frequency dependence of V959 Mon's major axis can be explained if the ejecta expanding along the major axis were becoming optically thin over the course of the imaging campaign.

30. Chomiuk, L. et al. Dramatic brightening of Nova Mon 2012 at high radio frequencies. Astron. Telegram 4352 (2012)

31. Napier, P. J., Thompson, R. \& Ekers, R. D. The Very Large Array—design and performance of a modern synthesis radio telescope. Proc. IEEE 71, 1295-1320 (1983).

32. McMullin, J. P., Waters, B., Schiebel, D., Young, W. \& Golap, K. in Astronomical Data Analysis Software and Systems XVI (eds Shaw, R. A., Hill, F. \& Bell, D. J.) 127-130 (ASP Conf. Ser. 376, Astronomical Society of the Pacific, 2007).

33. Greisen, E. W. in Information Handling in Astronomy-Historical Vistas (ed. Heck, A.) 109-125 (Astrophys. Space Sci. Library 285, Springer, 2003).

34. Shepherd, M. C., Pearson, T. J. \& Taylor, G. B. DIFMAP: an interactive program for synthesis imaging. Bull. Am. Astron. Soc. 26, 987-989 (1994)

35. Briggs, D. S. High fidelity interferometric imaging: robust weighting and NNLS deconvolution. Bull. Am. Astron. Soc. 27, 1444 (1995).

36. Sault, R. J., Teuben, P. J. \& Wright, M. C. H. in Astronomical Data Analysis Software and Systems IV (eds Shaw, R. A., Payne, H. E. \& Hayes, J. J. E.) 433-436 (ASP Conf. Ser. 77, Astronomical Society of the Pacific, 1995).

37. Fuhrmann, L. et al. Follow-up radio observations of Nova Mon 2012 at 10-142 GHz. Astron. Telegram 4376 (2012).

38. O'Brien, T. J. et al. Nova Mon 2012 resolved as a double radio source. Astron Telegram 4408 (2012). 


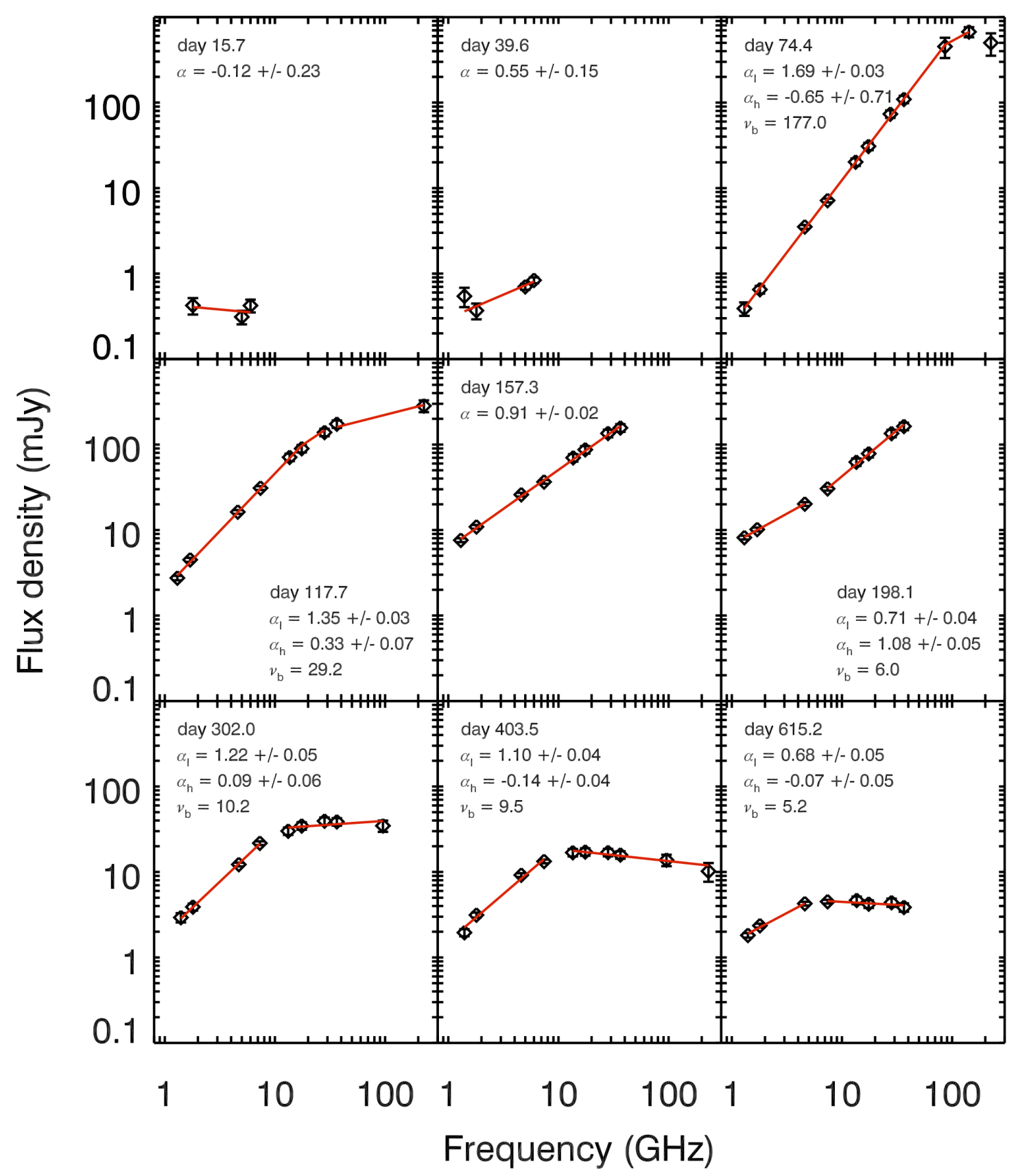

Extended Data Figure $1 \mid$ Radio/millimetre spectral evolution of V959 Mon. Measurements and $1 \sigma$ uncertainties from select epochs are shown as black points. Power-law or broken power-law fits are overplotted as red lines (the

function is chosen to minimize the reduced $\chi^{2}$ value). The best-fit spectral indices are listed in each panel, along with the break frequency $\left(v_{\mathrm{b}}\right)$ in the case of broken power-law fits. 


\section{RESEARCH LETTER}

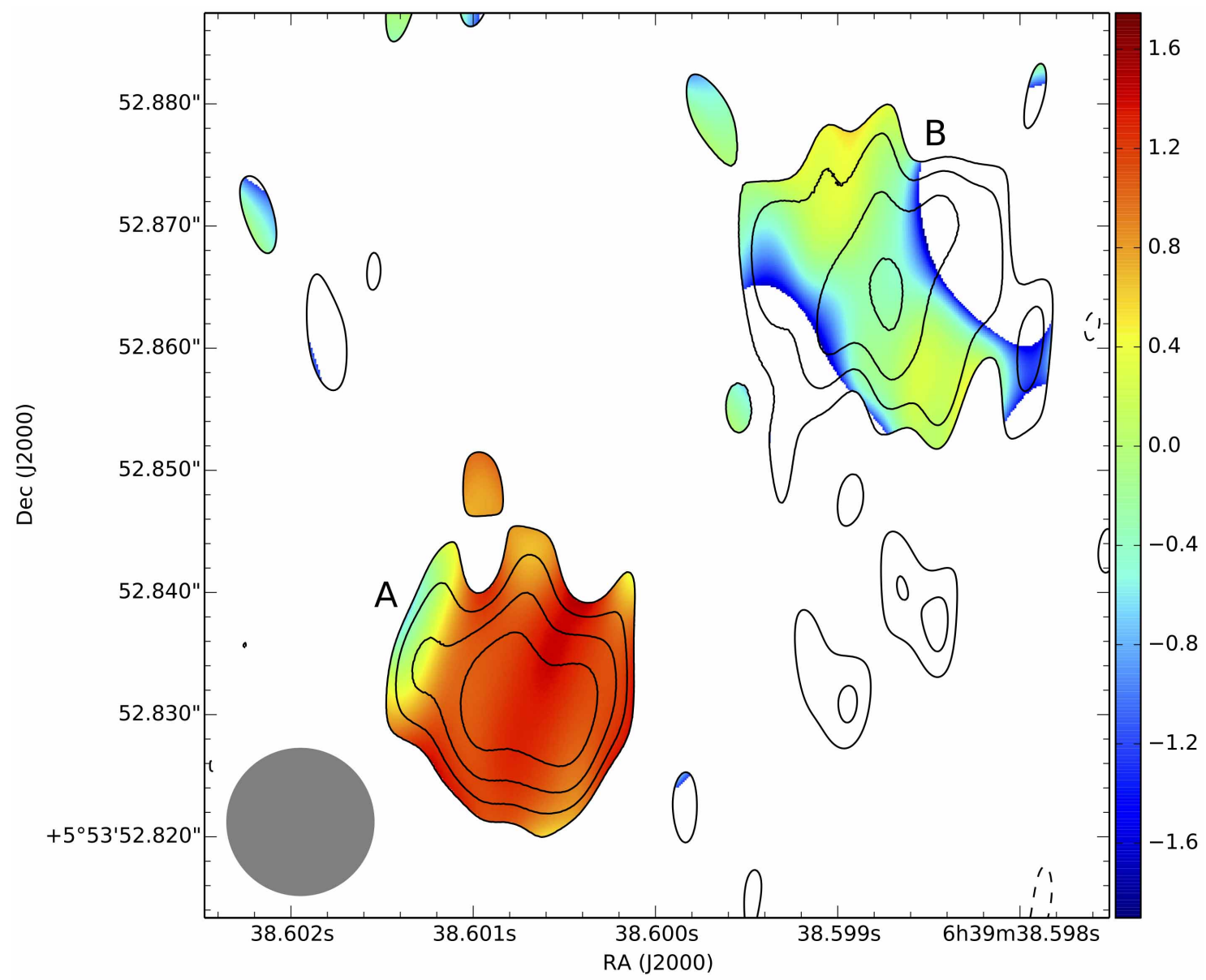

Extended Data Figure $2 \mid$ Spectral index map from 2012 October 3 VLBA observations. The spectral index is measured by comparing images at 1.6 and 5 GHz. Overlaid contours are from the $1.6 \mathrm{GHz}$ Stokes $I$ map. Contour levels are $-0.08,0.08,0.13,0.16,0.23,0.32$ and $0.45 \mathrm{mJy}$ per beam. 


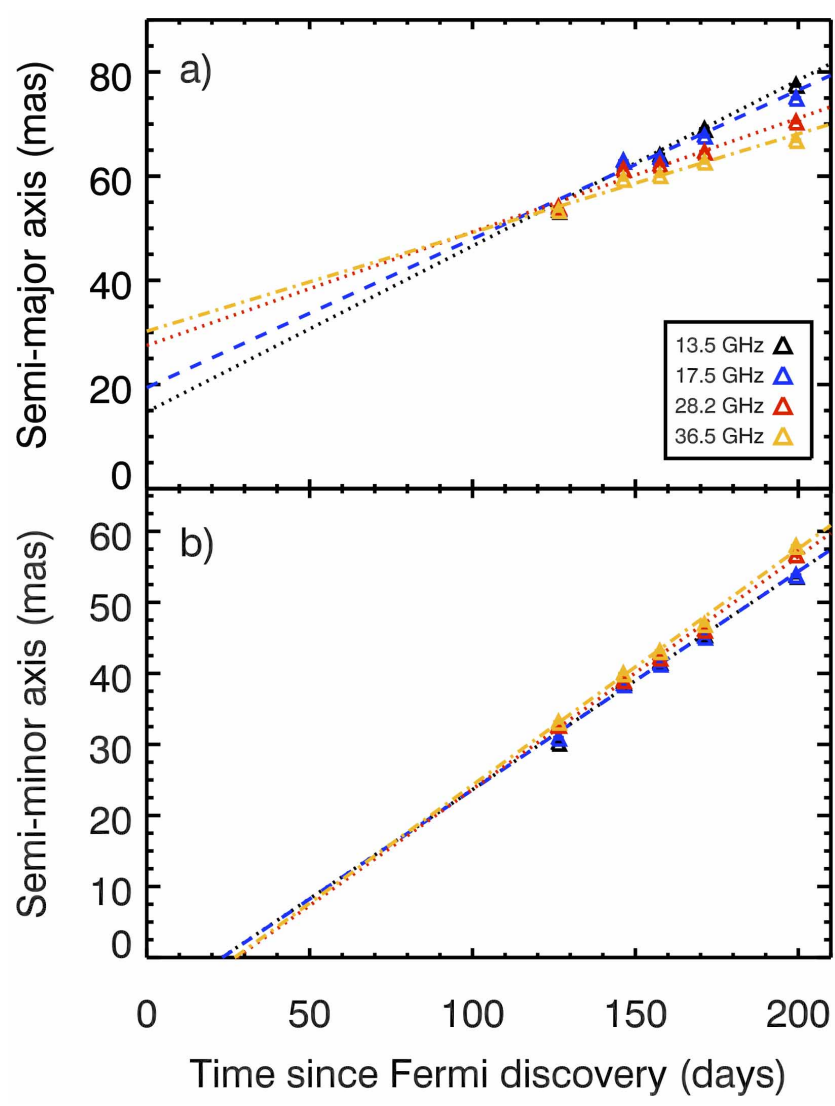

Extended Data Figure 3 The expansion of V959 Mon as a function of time. Semi-major axis (a) and semi-minor axis (b), both in units of milliarcseconds. Measurements at four distinct frequencies are plotted in different colours (see key). Error bars from JMFIT $(1 \sigma)$ are so small that they are not visible. Linear fits are made to each frequency separately, and are plotted as coloured lines. 


\section{RESEARCH LETTER}

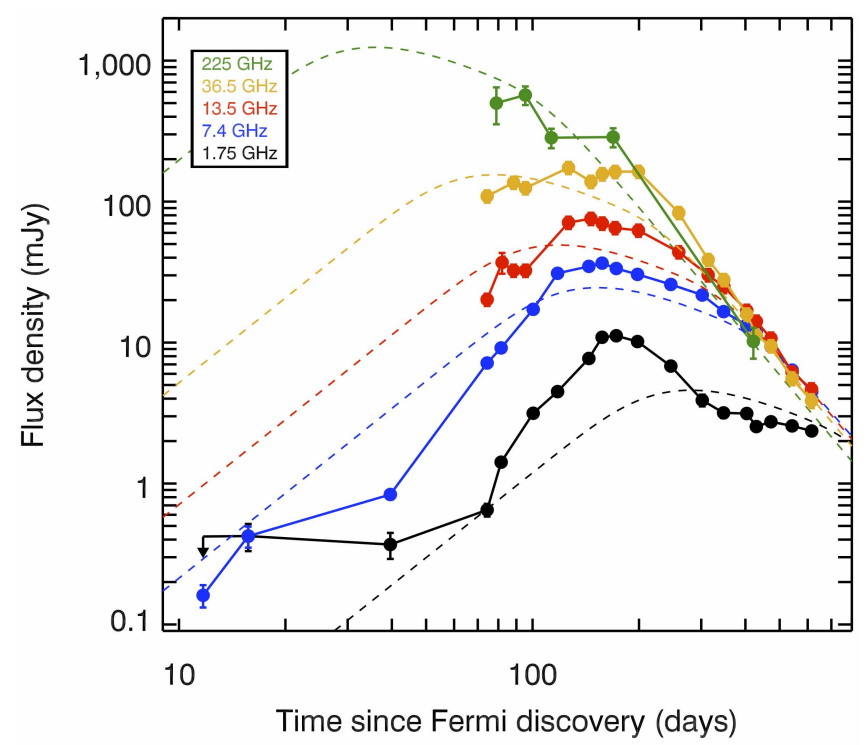

Extended Data Figure $4 \mid$ Model fit to the radio/millimetre light curve of V959 Mon. A simple model of thermal expanding ejecta roughly describes the light curve evolution at day $\sim 200$ and later, and implies an ejected mass of few $10^{-5}$ solar masses. Error bars denote $1 \sigma$ uncertainty. 
Extended Data Table 1 | VLA observations of V959 Mon

\begin{tabular}{|c|c|c|c|c|c|c|c|c|c|c|}
\hline $\begin{array}{l}\text { Date } \\
\text { (UT) }\end{array}$ & $\begin{array}{l}t-t_{0} \\
\text { (Days) }\end{array}$ & Conf. & $\begin{array}{c}\nu \\
(\mathrm{GHz})\end{array}$ & $\begin{array}{c}S_{\nu} \\
\text { (mJy) }\end{array}$ & $\begin{array}{c}\nu \\
(\mathrm{GHz})\end{array}$ & $\begin{array}{c}S_{\nu} \\
\text { (mJy) }\end{array}$ & $\begin{array}{c}\nu \\
(\mathrm{GHz})\end{array}$ & $\begin{array}{c}S_{\nu} \\
(\mathrm{mJy})\end{array}$ & $\begin{array}{c}\nu \\
(\mathrm{GHz})\end{array}$ & $\begin{array}{c}S_{\nu} \\
(\mathrm{mJy})\end{array}$ \\
\hline 2012 Jun 30.7 & 11.7 & B & 1.3 & $0.10 \pm 0.18$ & 1.8 & $0.15 \pm 0.09$ & 5.0 & $0.26 \pm 0.03$ & 6.0 & $0.16 \pm 0.03$ \\
\hline $2012 \mathrm{Jul} 4.7$ & 15.7 & B & 1.3 & $0.11 \pm 0.20$ & 1.8 & $0.42 \pm 0.09$ & 5.0 & $0.31 \pm 0.06$ & 6.0 & $0.42 \pm 0.07$ \\
\hline $2012 \mathrm{Jul} 28.6$ & 39.6 & B & 1.4 & $0.54 \pm 0.14$ & 1.8 & $0.37 \pm 0.08$ & 5.0 & $0.70 \pm 0.06$ & 6.0 & $0.84 \pm 0.07$ \\
\hline 2012 Sep 1.4 & 74.4 & A & $\begin{array}{c}1.3 \\
13.3\end{array}$ & $\begin{array}{c}0.39 \pm 0.07 \\
20.20 \pm 2.02\end{array}$ & $\begin{array}{c}1.8 \\
17.4\end{array}$ & $\begin{array}{c}0.65 \pm 0.07 \\
30.82 \pm 3.08\end{array}$ & $\begin{array}{c}4.6 \\
27.5\end{array}$ & $\begin{array}{c}3.52 \pm 0.18 \\
73.59 \pm 7.36\end{array}$ & $\begin{array}{c}7.4 \\
36.5\end{array}$ & $\begin{array}{c}7.16 \pm 0.36 \\
109.3 \pm 10.9\end{array}$ \\
\hline 2012 Sep 8.5 & 81.5 & $\mathrm{BnA}$ & 1.3 & $1.24 \pm 0.10$ & 1.8 & $1.42 \pm 0.10$ & 4.6 & $4.74 \pm 0.24$ & 7.4 & $9.16 \pm 0.46$ \\
\hline 2012 Sep 15.4 & 88.4 & $\mathrm{BnA}$ & $\begin{array}{l}13.5 \\
40.6\end{array}$ & $\begin{array}{l}32.46 \pm 3.25 \\
211.7 \pm 21.2\end{array}$ & $\begin{array}{l}17.4 \\
45.4\end{array}$ & $\begin{array}{l}47.25 \pm 4.73 \\
235.8 \pm 23.6\end{array}$ & 28.2 & $102.6 \pm 10.3$ & 36.5 & $136.6 \pm 13.7$ \\
\hline $2012 \operatorname{Sep} 22.5$ & 95.5 & $\mathrm{BnA}$ & 13.5 & $32.44 \pm 3.26$ & 17.4 & $61.76 \pm 6.25$ & 28.5 & $96.15 \pm 9.66$ & 36.5 & $124.9 \pm 12.6$ \\
\hline 2012 Sep 27.4 & 100.4 & $\mathrm{BnA} \rightarrow \mathrm{A}$ & 1.3 & $2.55 \pm 0.14$ & 1.8 & $3.15 \pm 0.17$ & 4.6 & $9.00 \pm 0.45$ & 7.4 & $17.21 \pm 0.88$ \\
\hline 2012 Oct 14.7 & 117.7 & A & 1.3 & $2.75 \pm 0.16$ & 1.7 & $4.49 \pm 0.24$ & 4.6 & $16.28 \pm 0.82$ & 7.4 & $30.99 \pm 1.57$ \\
\hline 2012 Oct 23.4 & 126.4 & A & 13.6 & $71.03 \pm 7.10$ & 17.5 & $89.70 \pm 8.97$ & 28.2 & $138.9 \pm 13.9$ & 36.5 & $173.9 \pm 17.4$ \\
\hline 2012 Nov 10.3 & 144.3 & A & 1.3 & $5.06 \pm 0.38$ & 1.8 & $7.71 \pm 0.48$ & 4.6 & $21.45 \pm 1.36$ & 7.4 & $34.73 \pm 1.78$ \\
\hline 2012 Nov 12.3 & 146.3 & A & 13.5 & $75.70 \pm 7.60$ & 17.5 & $90.08 \pm 9.03$ & 28.2 & $116.9 \pm 11.9$ & 36.5 & $138.6 \pm 14.0$ \\
\hline 2012 Nov 23.4 & 157.4 & A & $\begin{array}{c}1.3 \\
13.6\end{array}$ & $\begin{array}{c}7.61 \pm 0.39 \\
70.15 \pm 7.06\end{array}$ & $\begin{array}{c}1.8 \\
17.5\end{array}$ & $\begin{array}{l}10.90 \pm 0.55 \\
87.11 \pm 8.74\end{array}$ & $\begin{array}{c}4.6 \\
28.2\end{array}$ & $\begin{array}{l}25.94 \pm 1.30 \\
135.3 \pm 14.0\end{array}$ & $\begin{array}{c}7.4 \\
36.5\end{array}$ & $\begin{array}{l}36.60 \pm 1.83 \\
156.9 \pm 16.1\end{array}$ \\
\hline 2012 Dec 7.2 & 171.2 & A & 13.5 & $65.08 \pm 6.51$ & 17.5 & $80.92 \pm 8.09$ & 28.2 & $133.5 \pm 13.4$ & 36.5 & $163.0 \pm 16.3$ \\
\hline 2012 Dec 8.5 & 172.5 & A & 1.3 & $8.40 \pm 0.43$ & 1.8 & $11.17 \pm 0.56$ & 4.6 & $23.76 \pm 1.19$ & 7.4 & $33.51 \pm 1.68$ \\
\hline 2013 Jan 3.1 & 198.1 & A & 1.3 & $8.16 \pm 0.41$ & 1.7 & $10.16 \pm 0.51$ & 4.6 & $20.17 \pm 1.01$ & 7.4 & $30.45 \pm 1.52$ \\
\hline 2013 Jan 4.3 & 199.3 & A & 13.5 & $62.45 \pm 6.25$ & 17.5 & $78.38 \pm 7.84$ & 28.2 & $134.9 \pm 13.5$ & 36.5 & $163.4 \pm 16.4$ \\
\hline 2013 Feb 20.1 & 246.1 & $\mathrm{D}$ & 1.4 & $6.22 \pm 0.35$ & 1.8 & $6.81 \pm 0.36$ & 4.6 & $15.75 \pm 0.79$ & 7.4 & $25.83 \pm 1.29$ \\
\hline 2013 Mar 05.1 & 259.1 & $\mathrm{D}$ & 13.6 & $43.87 \pm 4.39$ & 17.5 & $54.63 \pm 5.46$ & 28.2 & $74.11 \pm 7.41$ & 36.5 & $83.35 \pm 8.34$ \\
\hline 2013 Apr 17.0 & 302.0 & $\mathrm{D}$ & 1.4 & $2.93 \pm 0.36$ & 1.8 & $3.90 \pm 0.37$ & 4.7 & $12.23 \pm 0.62$ & 7.3 & $21.73 \pm 1.09$ \\
\hline 2013 Apr 28.9 & 313.9 & $\mathrm{D}$ & 13.2 & $30.12 \pm 3.01$ & 17.5 & $34.66 \pm 3.47$ & 28.2 & $39.48 \pm 3.95$ & 36.5 & $38.69 \pm 3.87$ \\
\hline 2013 Jun 1.4 & 347.4 & $\mathrm{DnC} \rightarrow \mathrm{C}$ & $\begin{array}{c}1.4 \\
13.2\end{array}$ & $\begin{array}{c}2.69 \pm 0.37 \\
24.86 \pm 2.49\end{array}$ & $\begin{array}{c}1.7 \\
17.5\end{array}$ & $\begin{array}{c}3.17 \pm 0.26 \\
27.01 \pm 2.70\end{array}$ & $\begin{array}{c}4.6 \\
28.2\end{array}$ & $\begin{array}{l}10.53 \pm 0.53 \\
29.16 \pm 2.92\end{array}$ & $\begin{array}{c}7.4 \\
36.5\end{array}$ & $\begin{array}{l}16.58 \pm 0.83 \\
27.87 \pm 2.79\end{array}$ \\
\hline 2013 Jun 27.6 & 403.6 & $\mathrm{C}$ & $\begin{array}{c}1.4 \\
13.5\end{array}$ & $\begin{array}{c}1.95 \pm 0.19 \\
16.86 \pm 1.69\end{array}$ & $\begin{array}{c}1.8 \\
17.5\end{array}$ & $\begin{array}{c}3.14 \pm 0.21 \\
17.20 \pm 1.72\end{array}$ & $\begin{array}{c}4.6 \\
28.2\end{array}$ & $\begin{array}{c}9.21 \pm 0.46 \\
16.94 \pm 1.70\end{array}$ & $\begin{array}{c}7.4 \\
36.5\end{array}$ & $\begin{array}{l}13.30 \pm 0.67 \\
15.87 \pm 1.59\end{array}$ \\
\hline 2013 Aug 22.5 & 429.5 & $\mathrm{C}$ & $\begin{array}{c}1.4 \\
13.5\end{array}$ & $\begin{array}{c}1.81 \pm 0.23 \\
14.13 \pm 1.41\end{array}$ & $\begin{array}{c}1.8 \\
17.5\end{array}$ & $\begin{array}{c}2.53 \pm 0.18 \\
14.11 \pm 1.41\end{array}$ & $\begin{array}{c}4.6 \\
28.2\end{array}$ & $\begin{array}{c}8.72 \pm 0.44 \\
13.09 \pm 1.32\end{array}$ & $\begin{array}{c}7.4 \\
36.5\end{array}$ & $\begin{array}{l}11.94 \pm 0.60 \\
11.27 \pm 1.14\end{array}$ \\
\hline 2013 Oct 04.4 & 472.4 & B & $\begin{array}{c}1.4 \\
13.6\end{array}$ & $\begin{array}{c}1.96 \pm 0.14 \\
10.71 \pm 1.07\end{array}$ & $\begin{array}{c}1.8 \\
17.5\end{array}$ & $\begin{array}{c}2.74 \pm 0.16 \\
10.53 \pm 1.05\end{array}$ & $\begin{array}{c}4.6 \\
28.2\end{array}$ & $\begin{array}{c}7.60 \pm 0.38 \\
10.11 \pm 1.01\end{array}$ & $\begin{array}{c}7.4 \\
36.5\end{array}$ & $\begin{array}{l}9.38 \pm 0.47 \\
9.45 \pm 0.96\end{array}$ \\
\hline 2013 Dec 13.2 & 542.2 & B & $\begin{array}{c}1.4 \\
13.6\end{array}$ & $\begin{array}{l}1.88 \pm 0.17 \\
6.16 \pm 0.62\end{array}$ & $\begin{array}{c}1.8 \\
17.5\end{array}$ & $\begin{array}{l}2.56 \pm 0.15 \\
6.60 \pm 0.66\end{array}$ & $\begin{array}{c}4.6 \\
28.2\end{array}$ & $\begin{array}{l}5.76 \pm 0.29 \\
6.33 \pm 0.64\end{array}$ & $\begin{array}{c}7.4 \\
36.5\end{array}$ & $\begin{array}{l}6.38 \pm 0.32 \\
5.56 \pm 0.59\end{array}$ \\
\hline $2014 \mathrm{Feb} 24.2$ & 615.2 & A & 13.6 & $4.67 \pm 0.48$ & 17.5 & $4.23 \pm 0.43$ & 28.2 & $4.39 \pm 0.48$ & 36.5 & $3.86 \pm 0.43$ \\
\hline 2014 Feb 25.0 & 616.0 & A & 1.4 & $1.82 \pm 0.11$ & 1.8 & $2.36 \pm 0.12$ & 4.6 & $4.27 \pm 0.21$ & 7.4 & $4.50 \pm 0.23$ \\
\hline
\end{tabular}




\section{RESEARCH LETTER}

Extended Data Table 2 | Millimetre observations of V959 Mon

\begin{tabular}{ccccc}
\hline \hline $\begin{array}{c}\text { Date } \\
\text { (UT) }\end{array}$ & $\begin{array}{c}t-t_{0} \\
\text { (Days) }\end{array}$ & Facility & $\begin{array}{c}\nu \\
(\mathrm{GHz})\end{array}$ & $\begin{array}{c}S_{\nu} \\
(\mathrm{mJy})\end{array}$ \\
\hline 2012 Sep 6 & 79 & IRAM $^{38}$ & 86 & $453 \pm 121$ \\
2012 Sep 6 & 79 & SMA & 225 & $500 \pm 125$ \\
2012 Sep 8 & 81 & PdBI & 87 & $330 \pm 33$ \\
2012 Sep 22 & 95 & SMA & 225 & $570 \pm 57$ \\
2012 Oct 10 & 113 & SMA & 225 & $284 \pm 28$ \\
2012 Dec 5 & 169 & SMA & 220 & $287 \pm 28$ \\
2013 May 21 & 336 & CARMA & 96 & $34.7 \pm 3.6$ \\
2013 Aug 11 & 418 & CARMA & 96 & $13.8 \pm 1.5$ \\
2013 Aug 14 & 421 & CARMA & 230 & $10.2 \pm 2.0$ \\
\hline
\end{tabular}

Flux density measurements obtained with the SMA, CARMA, IRAM and PdBI telescopes at 86-230 GHz. The time $t_{0}$ corresponds to $\gamma$-ray discovery, 2012 June $19^{5}$. 
Extended Data Table 3 | VLBI components of V959 Mon

\begin{tabular}{|c|c|c|c|c|c|c|c|}
\hline $\begin{array}{l}\text { Date } \\
\text { (UT) }\end{array}$ & $\begin{array}{l}t-t_{0} \\
\text { (Days) }\end{array}$ & Facility & $\begin{array}{c}\nu \\
(\mathrm{GHz})\end{array}$ & $\begin{array}{c}S_{\nu} \\
(\mathrm{mJy})\end{array}$ & $\begin{array}{c}\text { RA Pos } \\
(06 \mathrm{~h} 39 \mathrm{mXXs})\end{array}$ & $\begin{array}{c}\text { Dec Pos } \\
\left(05 \mathrm{~d} 53^{\prime} \mathrm{XX}^{\prime \prime}\right)\end{array}$ & $\begin{array}{c}\text { Phase Ref. } \\
\text { Source }\end{array}$ \\
\hline \multicolumn{8}{|c|}{ Component A } \\
\hline 2012 Sep 18 & 91 & EVN & 5.0 & $1.04 \pm 0.11$ & 38.60057 & 52.8267 & J0645+0541 \\
\hline 2012 Oct 03 & 106 & VLBA & 1.6 & $0.53 \pm 0.06$ & 38.60077 & 52.8311 & $\mathrm{~J} 0650+0358$ \\
\hline 2012 Oct 03 & 106 & VLBA & 5.0 & $1.66 \pm 0.17$ & 38.60087 & 52.8315 & $\mathrm{~J} 0650+0358$ \\
\hline 2012 Oct 10 & 113 & EVN & 5.0 & $1.45 \pm 0.15$ & 38.60077 & 52.8225 & J0645+0541 \\
\hline 2012 Oct 14 & 117 & VLBA & 1.6 & $0.17 \pm 0.05$ & 38.60054 & 52.7915 & $\mathrm{~J} 0650+0358$ \\
\hline 2012 Oct 14 & 117 & VLBA & 5.0 & $1.31 \pm 0.14$ & 38.60097 & 52.8327 & $\mathrm{~J} 0650+0358$ \\
\hline 2012 Oct 30 & 133 & VLBA & 1.6 & $1.20 \pm 0.13$ & 38.60092 & 52.82778 & J0645+0541 \\
\hline 2012 Oct 30 & 133 & VLBA & 5.0 & $1.03 \pm 0.12$ & 38.60098 & 52.82325 & $\mathrm{~J} 0650+0358$ \\
\hline \multicolumn{8}{|c|}{ Component B } \\
\hline 2012 Sep 18 & 91 & EVN & 5.0 & $0.42 \pm 0.05$ & 38.59886 & 52.8514 & $\mathrm{~J} 0645+0541$ \\
\hline 2012 Oct 03 & 106 & VLBA & 1.6 & $0.59 \pm 0.07$ & 38.59883 & 52.8668 & $\mathrm{~J} 0650+0358$ \\
\hline 2012 Oct 03 & 106 & VLBA & 5.0 & $0.16 \pm 0.03$ & 38.59881 & 52.8597 & $\mathrm{~J} 0650+0358$ \\
\hline 2012 Oct 10 & 113 & EVN & 5.0 & $0.31 \pm 0.04$ & 38.59852 & 52.8555 & J0645+0541 \\
\hline 2012 Oct 14 & 117 & VLBA & 1.6 & $0.52 \pm 0.07$ & 38.59865 & 52.8620 & $\mathrm{~J} 0650+0358$ \\
\hline 2012 Oct 14 & 117 & VLBA & 5.0 & $0.16 \pm 0.04$ & 38.59867 & 52.8626 & $\mathrm{~J} 0650+0358$ \\
\hline \multicolumn{8}{|c|}{ Component $\mathrm{C}$} \\
\hline 2012 Oct 10 & 113 & EVN & 5.0 & $0.36 \pm 0.05$ & 38.60251 & 52.8518 & J0645+0541 \\
\hline 2012 Oct 14 & 117 & VLBA & 5.0 & $0.14 \pm 0.04$ & 38.60267 & 52.8622 & $\mathrm{~J} 0650+0358$ \\
\hline
\end{tabular}

Positions and flux densities of VLBI knots, observed with EVN and VLBA. The time to corresponds to $\gamma$-ray discovery, 2012 June $19^{5}$. 